\title{
Kids, Adolescents, and Young Adults \\ Cancer Study_A Methodologic Approach in Cancer Epidemiology Research
}

\author{
Nancy J. Link, ${ }^{1}$ Eva Maurer, ${ }^{1}$ Joan Largent, ${ }^{1}$ Erin Kent, ${ }^{1}$ Rebecca A. Morris, ${ }^{2}$ \\ Leonard S. Sender, ${ }^{1,2,3}$ and Hoda Anton-Culver ${ }^{1}$ \\ ${ }^{1}$ Department of Epidemiology, School of Medicine, University of California, Irvine, 224 Irvine Hall, Irvine, CA 92697, USA \\ ${ }^{2}$ CHOC Children's Hospital, 455 S. Main Street, Orange, CA 92868, USA \\ ${ }^{3}$ Chao Family Comprehensive Cancer Center, University of California, Irvine, 101 The City Drive South, Bldg 56, Room 205, \\ Orange, CA 92868, USA
}

Correspondence should be addressed to Hoda Anton-Culver, hantoncu@uci.edu

Received 26 August 2009; Accepted 13 November 2009

Recommended by Lidia Larizza

Advances have been made in treatment and outcomes for pediatric cancer. However adolescents and young adults (AYAs) with cancer have not experienced similar relative improvements. We undertook a study to develop the methodology necessary for epidemiologic cancer research in these age groups. Our goal was to create the Kids, Adolescents, and Young Adults Cancer (KAYAC) project to create a resource to address research questions relevant to this population. We used a combination of clinic and population-based ascertainment to enroll 111 cases aged 0-39 for this methodology development study. The largest groups of cancer types enrolled include: breast cancer, leukemia, lymphoma, and melanoma. The overall participation rate is $69.8 \%$ and varies by age and tumor type. The study included patients, mothers, and fathers. The methods used to establish this resource are described, and the values of the resource in studies of childhood and young adult cancer are outlined.

Copyright (C) 2009 Nancy J. Link et al. This is an open access article distributed under the Creative Commons Attribution License, which permits unrestricted use, distribution, and reproduction in any medium, provided the original work is properly cited.

\section{Introduction}

Childhood, adolescent, and young adult cancers are among the "rare cancers" and consequently very few epidemiologic studies are carried out to address research questions related to etiology, clinical outcome, and disparities in this group of patients (0-39 years of age). Further, studies of adolescent and young adult (AYA) cancer (age 1539) are less frequent compared to studies of childhood cancer (0-14 years). Multiple reasons may account for this deficiency including: (1) low incidence rates particularly in certain cancers; (2) difficulties in obtaining and assessing risk factor information especially in young patients; (3) lack of standardized health care access for AYA patients; (4) diagnosis and treatment often rendered by pediatric or adult oncologists rather than AYA specialized oncologists; (5) lack of fully developed methodology for these age groups $[1,2]$.
It is clear that the mortality rate due to cancer in children, adolescents, and young adults is variable by age, even within the same type of cancer. In addition, adolescents and young adults are at a higher risk for different types of cancers compared to younger children, such as lymphomas, bone tumors, and soft-tissue sarcomas [3]. The disparity that results from these differences is particularly seen by race/ethnicity, in terms of the cancer diagnosis, access to treatment, eligibility for the same clinical trials and care [4].

In 2006 the National Cancer Institute (NCI) and the Lance Armstrong Foundation convened a Progress Review Group (PRG) of experts in the area of Adolescent and Young Adult Oncology (AYAO) and issued a report describing the research and care imperatives for cancer in the AYA population [1]. While significant advances have been made in treatment and outcomes for pediatric patients, the PRG reported that AYAs have not experienced similar relative improvements [1]. Several interrelated factors may 
contribute to the lack of improvement in early detection, diagnosis, treatment, outcome, and follow-up care including: (a) lack of reliable data and deficiency of research specific for the AYA population, (b) increased sense of personal invincibility prevalent in the AYA population that influences their clinical outcomes, (c) low suspicion of cancer diagnosis in this age group by health providers, (d) a lack of adequate health insurance (AYAs) (have the highest uninsured rate of any age group in the US) [5], (e) lack of primary care and oncology infrastructure focused specifically on AYA health, and (f) poor understanding of patient and tumor biology. In addition AYAs are rarely referred to the few clinical trials available for their age group which can result in use of treatment protocols that have not been specifically tested in adolescent or young adult cancer cases [3, 4].

The PRG identified five research and care imperatives for improving the outcomes of adolescents and young adults with cancer, one of which described the need to create the tools necessary to study the AYA cancer problem in particular to (1) create a prospective database on all AYA cancer patients, (2) increase the number of annotated tumor, normal tissue, and other biospecimens, and (3) create or modify assessment tools specific to AYA cancer issues $[1,6]$.

In response to the PRG recommendations, we undertook a study to develop the methodology necessary for epidemiologic studies of cancer in these age groups and included children aged 0-14 to allow for comparisons of the experience between childhood and AYA participants and to offer recommendations for future epidemiologic studies in these age groups.

The research goal of KAYAC is to develop protocols and collect risk factor and clinical data and biospecimens from kids, adolescents, and young adults with cancer to be used to investigate: (a) whether adolescent and young adult cancers are different from childhood and older adult cancers with respect to specific genetic susceptibility to exposures, etiologic risk factors, and whether they can be characterized using biological markers; (b) the degree of aggressiveness of cancer in adolescents and young adults and the cancer outcome in AYA patients; (c) examine barriers to health care access using questionnaire data collected from AYAs and their parents. This resource is envisioned to be used in future studies for planning cancer prevention and control in this unique population.

\section{Materials and Methods}

2.1. Study Design and Protocol. We developed the study protocol as outlined in Figure 1. After protocol development, Institutional Review Board approval was obtained at UC Irvine (HS\# 2006-4958) and CHOC Children's Hospital (IRB\# 070538).

Three versions of study questionnaires were developed using previously validated questions; one each for cases, mothers and fathers. Questionnaires were pilot tested, and finalized versions were deployed into the field. Items included in the questionnaires were designed to obtain data from the parents on exposures up to 1 year preconception, during pregnancy and after birth to the date of diagnosis. We also collected risk factor information, family history of cancer as well as health history information from the case and their parents. Additional questionnaire items included immunization and general environmental exposures, residential history, parental occupational exposures, and a psychosocial needs assessment. Family cancer history information was also used to generate picture pedigrees and verification tables that were mailed to subjects for confirmation and corrections.

In addition to questionnaire data, clinical variables were also obtained from the cancer registry such as date of diagnosis, tumor information (site, histology, behavior, and differentiation), disease stage, and treatment history. Demographic variables were collected from both the cancer registry and the study questionnaires. Linkage with the population-based cancer registry provides valuable followup information including vital status, subsequent primary tumor diagnoses, date of last follow-up or death, and cause of death. Table 1 provides a summary of collected data items. The questionnaires used are available upon request.

For cases with no mother or father available, we interviewed legally authorized representatives (LARs). In some cases, where only the mother or the father was available, we interviewed one parent in addition to the cancer patient (over age 15). For children under age 15, the parent/LAR completed the patient questionnaire on the case's behalf. Blood samples were collected from patients (aged 0-14) at the pediatric clinic and from their parent(s)/LAR at their convenience, at the clinic by hospital staff or home by a certified phlebotomist. For patients aged 15-39 and their parent(s)/LAR, blood was collected either in their home or at our research facility. Participants and/or parents living outside of the three-county recruitment area were sent a kit containing appropriate specimen tubes and specific instructions for a laboratory or doctor's office to collect the sample and return to us by overnight delivery mail using provided prepaid shipping labels and packaging. All blood samples received barcode labels before being processed in the Department of Epidemiology laboratory, where all barcode labeled aliquots were tracked using the KAYAC study database.

2.2. Eligibility. Eligible patients were those diagnosed with cancer between 1/1/2006 and 6/30/2008 and their parents/LAR. Patients were ascertained through the populationbased cancer registry. Patients below age 15 were first contacted through collaboration with the CHOC Children's Hospital. Patients between 15 and 39 were contacted using information from the cancer registry. For all patients the methodology below was used by the study staff in the UCI Epidemiology Department.

\subsection{Ascertainment and Recruitment}

2.3.1. Childhood Cases Aged 0-14. Patients and their parent(s)/LAR were approached by a physician or other clinic staff during the patient's regular clinic visit and they were 


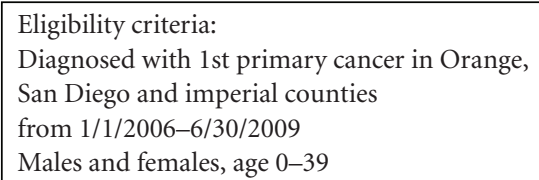

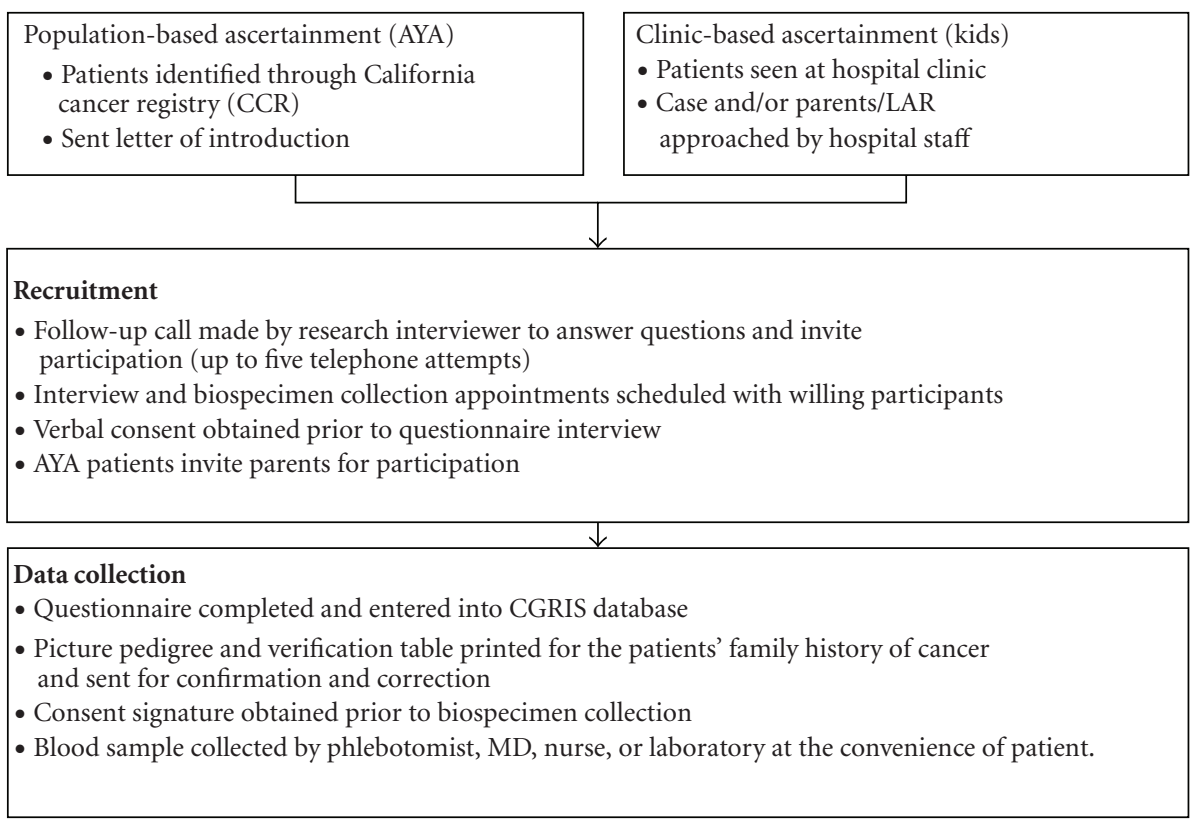

FIgURE 1: Study protocol.

invited to participate. If they were willing to participate, the clinic staff asked that the parent sign their approval for contact information to be provided to the KAYAC research office. Upon receipt of the signed contact information sheet, the Epidemiology Department study recruiter contacted the family, described the study including eligibility criteria, research procedures, and length of time required to complete participation and scheduled a time and place for the interview and blood draw for the parents. Patients were scheduled for blood draw during a regular clinic appointment. We developed and applied this protocol in the recruitment of 44 patients as a pilot study. Descriptive data on these 44 patients is presented in Table 2 .

2.3.2. Adolescent and Young Adult Cases Aged 15-39. Eligible patients were mailed a recruitment packet which included a letter of introduction that briefly described the study, eligibility criteria, research procedures, and estimated length of time required to complete participation. Also included in the packet were the KAYAC study brochure and a contact/interest reply form with a postage-paid return envelope. By using the contact/interest reply form, subjects were able to provide their preferred method of contact (i.e., email, home, or mobile phone), best day and time to reach them, or to decline participation. Two weeks after packets were mailed, an interviewer contacted the patient by telephone to answer any questions, and if the patient was willing, enroll them in the study. Those with Spanish surnames were mailed recruitment packet materials in both English and Spanish and contacted by a bilingual research associate. One or both parents/LAR of willing participants were recruited for study participation by invitation from the case. If the parent(s)/LAR agreed to participate, a KAYAC interviewer completed the enrollment protocol. We developed this protocol and applied it in the recruitment of 82 patients as a pilot study. Descriptive data on these 82 patients is presented in Table 2.

\subsubsection{Detailed Contact Procedures for All Participants, Aged} 0-39 Years. Up to five telephone attempts were made to contact each subject. A telephone "attempt" included leaving a message with a household member, leaving a voicemail message, or calling without leaving a message. After five telephone attempts resulting in no direct contact with the subject, a second recruitment packet was mailed. If all recruitment attempts proved to be unsuccessful, the subject file was closed and coded as unable to contact (UTC). Interviewers bilingual in English and Spanish were available to conduct all recruitment and research procedures.

All study participants were assigned unique identification numbers (family and member IDs) upon ascertainment. The study ID numbers were used on all study documents, database tracking screens, and biospecimen samples to ensure confidentiality of patient information. Subject personal identifiers are never included in analysis, reporting, or publications. Participants and parents were assured that they 
TABLE 1: Representative sample of variables collected in KAYAC case and parent questionnaires.

\begin{tabular}{|c|c|c|c|c|c|c|c|c|}
\hline & $\begin{array}{l}\text { Case (birth-early } \\
\text { childhood) }\end{array}$ & Case & $\begin{array}{l}\text { Case (at } \\
\text { diagnosis) }\end{array}$ & Mother & $\begin{array}{l}\text { Mother } \\
\text { Prenatal }\end{array}$ & Father & $\begin{array}{c}\text { Father } \\
\text { Prenatal }\end{array}$ & $\begin{array}{c}\text { Extended } \\
\text { family }\end{array}$ \\
\hline \multicolumn{9}{|l|}{ Demographics } \\
\hline Race/ethnicity & & $\mathrm{X}$ & & $\mathrm{X}$ & & $\mathrm{X}$ & & $\mathrm{X}$ \\
\hline Marital status & & $\mathrm{X}$ & & $\mathrm{X}$ & & $\mathrm{X}$ & & \\
\hline Date of birth & & $\mathrm{X}$ & & $\mathrm{X}$ & $\mathrm{X}$ & $\mathrm{X}$ & $\mathrm{X}$ & \\
\hline Date of death (if applicable) & & & & $\mathrm{X}$ & & $\mathrm{X}$ & & $\mathrm{X}$ \\
\hline Religious affiliation & & $\mathrm{X}$ & & $\mathrm{X}$ & & $\mathrm{X}$ & & $\mathrm{X}$ \\
\hline Birthplace & & $\mathrm{X}$ & & $\mathrm{X}$ & & $\mathrm{X}$ & & $\mathrm{X}$ \\
\hline \multicolumn{9}{|l|}{ Cancer information } \\
\hline History of cancer & & $\mathrm{X}$ & & $\mathrm{X}$ & & $\mathrm{X}$ & & $\mathrm{X}$ \\
\hline Cancer type and histology & & $\mathrm{X}$ & & $\mathrm{X}$ & & $\mathrm{X}$ & & $\mathrm{X}$ \\
\hline Date/age of diagnosis & & $\mathrm{X}$ & & $\mathrm{X}$ & & $\mathrm{X}$ & & $\mathrm{X}$ \\
\hline Hospital of diagnosis & & $\mathrm{X}$ & & $\mathrm{X}$ & & $\mathrm{X}$ & & $\mathrm{X}$ \\
\hline \multicolumn{9}{|l|}{ Social and economic status (SES) } \\
\hline Education & $\mathrm{X}$ & $\mathrm{X}$ & & $\mathrm{X}$ & & $\mathrm{X}$ & & \\
\hline Health insurance & $\mathrm{X}$ & $\mathrm{X}$ & $\mathrm{X}$ & & & & & \\
\hline Income & & $\mathrm{X}$ & X (household) & & & & & \\
\hline \multicolumn{9}{|l|}{ Risks, exposures, general health } \\
\hline General Health and Illnesses & $\mathrm{X}$ & $\mathrm{X}$ & & $\mathrm{X}$ & $\mathrm{X}$ & $\mathrm{X}$ & $\mathrm{X}$ & $\mathrm{X}$ \\
\hline Height and weight & $\mathrm{X}$ & $\mathrm{X}$ & $\mathrm{X}$ & $\mathrm{X}$ & $\mathrm{X}$ & & & \\
\hline Immunizations & $\mathrm{X}$ & $\mathrm{X}$ & & & $\mathrm{X}$ & & & \\
\hline Medications and vitamins taken & $\mathrm{X}$ & $\mathrm{X}$ & & $\mathrm{X}$ & $\mathrm{X}$ & $\mathrm{X}$ & $\mathrm{X}$ & \\
\hline Pesticide and chemical exposure & $\mathrm{X}$ & $\mathrm{X}$ & & $\mathrm{X}$ & $\mathrm{X}$ & $\mathrm{X}$ & $\mathrm{X}$ & \\
\hline Radiation/X-ray exposure & $\mathrm{X}$ & $\mathrm{X}$ & & & $\mathrm{X}$ & & & \\
\hline Animal exposure & $\mathrm{X}$ & & & & $\mathrm{X}$ & & & \\
\hline International travel & & $\mathrm{X}$ & & & & & & \\
\hline Occupational history & & $\mathrm{X}$ & & $\mathrm{X}$ & $\mathrm{X}$ & $\mathrm{X}$ & $\mathrm{X}$ & \\
\hline Tobacco use & & $\mathrm{X}$ & & $\mathrm{X}$ & $\mathrm{X}$ & $\mathrm{X}$ & $\mathrm{X}$ & $\mathrm{X}$ \\
\hline Alcohol use & & $\mathrm{X}$ & & & $\mathrm{X}$ & & & \\
\hline Breastfeeding & $\mathrm{X}$ & & & & & & & \\
\hline Reproductive history & & $\mathrm{X}$ & & $\mathrm{X}$ & $\mathrm{X}$ & & & \\
\hline \multicolumn{9}{|l|}{ Other } \\
\hline Psychological/emotional well-being & & $\mathrm{X}$ & & & & & & \\
\hline Social support & & $\mathrm{X}$ & & & & & & \\
\hline Quality of life & & $\mathrm{X}$ & & & & & & \\
\hline
\end{tabular}

could refuse to participate in the study or in any portion of the study at any time without jeopardizing their medical care.

2.4. Data and Biospecimen Processing. Participant electronic data including biospecimen and subject tracking, questionnaire, and family cancer history information, were entered into the research database previously developed by the UCI Department of Epidemiology: the Cancer Genetic Research Information System (CGRIS). To protect the confidentiality of research participants, this central informatics system includes use of encryption software, a firewall system, password requirements for access, storage of confidential information in separate extract files, and study reporting system that contains no confidential information. Participant paper research files are stored in a large medical records room with controlled access behind locked doors and passkey protected entry.

Blood samples were barcode labeled prior to being processed into serum, plasma, red blood cells, DNA, lymphocytes, and buffy coats and stored in $-80^{\circ} \mathrm{C}$ or $-140^{\circ} \mathrm{C}$ freezers. All sample aliquots received a barcode label that was scanned into the CGRIS biospecimen tracking database and identifies the location of where the specimens are stored allowing for future retrieval and links each specimen to corresponding subject data. 
TABle 2: Participation by subject type.

\begin{tabular}{|c|c|c|c|}
\hline \multicolumn{2}{|l|}{ Children (age 0-14) } & \multicolumn{2}{|l|}{ AYA (age 15-39) } \\
\hline Total ascertained and agreed to be contacted & 44 & Total ascertained and contact was attempted & 218 \\
\hline Participated in the study & 29 & Participated in the study & 82 \\
\hline Agreed to be contacted but later declined participation & 15 & Unable to contact & 87 \\
\hline \multirow{2}{*}{ - } & - & Contacts were made but patient declined participation & 33 \\
\hline & & Deceased & 16 \\
\hline Participation rate & $65.9 \%$ & Participation rate (excluding deceased and UTC) & $71.3 \%$ \\
\hline \multicolumn{4}{|l|}{ Parents } \\
\hline Number of invited eligible parents & & 215 & \\
\hline Participated in the study & & 135 & \\
\hline Declined participation & & 46 & \\
\hline Deceased & & 9 & \\
\hline $\begin{array}{l}\text { No permission given by the patient to } \\
\text { contact the parent }\end{array}$ & & 25 & \\
\hline Participation rate (excluding deceased) & & $65.5 \%$ & \\
\hline
\end{tabular}

\section{Results and Discussion}

3.1. Childhood Cases Aged 0-14. As is shown in Table 2, in collaboration with the children's hospital we ascertained 44 childhood cancer cases whose parents agreed to be contacted by the study team for enrollment in the study. We determined that the invitation to participate be presented first to the patient and their family by the physician in order to increase the likelihood of participation and to ensure that any questions that parents may have about their child's participation would be answered by the child's doctor. This method of engaging the physician in the KAYAC study recruitment has been very successful and it increased our ability to obtain support and approval of the attending physicians.

To date 29 childhood cancer patients and one or both of their parents have participated by completing questionnaires and donating blood samples. It is of note that parents of an additional 15 patients who initially agreed to participate subsequently decided against participation at the time of contact by the research associate however, these families may decide to join the study in the future. The change in willingness to participate may be influenced by their child's clinical condition or by other factors including emotional burden or time commitments. To date, the overall participation rate for this age group is $65.9 \%$. Demographic distributions for the 29 participants are included in Table 3.

There were equal numbers of males and females and a majority (55.2\%) were Hispanic (Table 3). Figure 2 describes the distribution of patients by cancer type. The majority of the patients have leukemia, a total of 25 of whom 17 participated for a participation rate of $68 \%$. The participation rate for lymphoma was $80 \%$ and was lower for brain cancer and sarcoma; however there were a very small number of these cases.

3.2. Adolescent and Young Adult (AYA) Cases Aged 15-39. A total of 82 AYA cases were enrolled in the KAYAC study. The

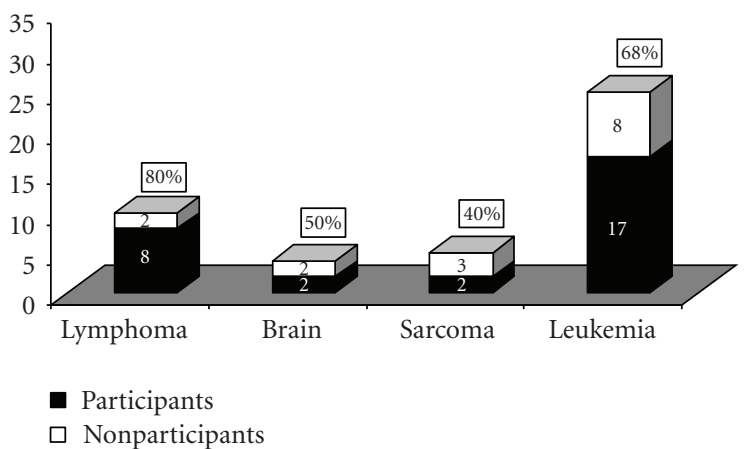

(a)

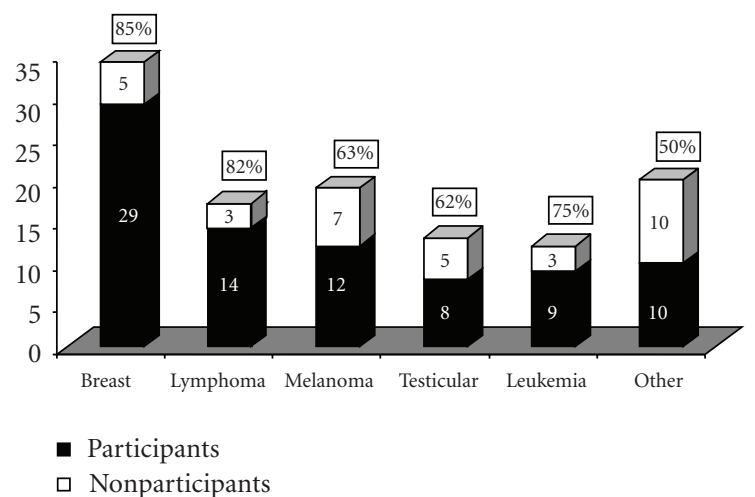

(b)

FIGURE 2: (a) Participation rate by cancer type for pediatric population (age 0-14). (b) Participation rate by cancer type for AYA population (age 15-39).

cancer-type distribution of the 82 enrolled cases is shown in Figure 2. The most common cancer types for AYA cases enrolled in the study were breast, leukemia, lymphoma, and melanoma. 
TAble 3: Patient Demographics.

\begin{tabular}{|c|c|c|c|c|c|}
\hline \multicolumn{3}{|c|}{$\begin{array}{l}\text { Children } 0-14 \text { years old } \\
\qquad(n=29)\end{array}$} & \multicolumn{3}{|c|}{$\begin{array}{c}\text { AYA } 15-39 \text { years old } \\
(n=82)\end{array}$} \\
\hline Sex & No. & $\%$ & Sex & No. & $\%$ \\
\hline male & 15 & $51.7 \%$ & male & 27 & $32.9 \%$ \\
\hline female & 14 & $48.3 \%$ & female & 55 & $67.0 \%$ \\
\hline Age & & & Age & & \\
\hline $0-5$ & 8 & $27.6 \%$ & $15-19$ & 6 & $7.3 \%$ \\
\hline $6-10$ & 10 & $34.5 \%$ & $20-24$ & 12 & $14.6 \%$ \\
\hline \multirow[t]{3}{*}{$11-14$} & 11 & $37.9 \%$ & $25-29$ & 23 & $28.0 \%$ \\
\hline & & & $30-34$ & 14 & $17.0 \%$ \\
\hline & & & $34-39$ & 27 & $32.9 \%$ \\
\hline Race/Ethnicity & & & Race/ethnicity & & \\
\hline Non-Hispanic white & 8 & $26.6 \%$ & Non-Hispanic white & 53 & $64.6 \%$ \\
\hline Hispanic & 16 & $55.2 \%$ & Hispanic & 23 & $28.0 \%$ \\
\hline Asian & 5 & $17.2 \%$ & Asian & 6 & $7.3 \%$ \\
\hline
\end{tabular}

Of 218 AYA cases identified as eligible in the cancer registry, 82 enrolled in KAYAC, yielding a $71.3 \%$ participation rate (Table 2 ) among a population often assumed to be difficult to access and reluctant to participate in research [7]. As shown in Figure 2, the cancer type with the highest rate of participation for the AYA group is breast (85\%) followed by lymphoma (82\%) and leukemia (75\%).

We believe that these participation rates are excellent for epidemiologic studies in AYA and encouraging for future application to cancer epidemiologic studies in this age group. The participation rate for parents was 65\% (Table 2). As described in the methods above we contacted the parent or parents only with permission of the AYA patient, and only after they have had an opportunity to describe the study to the parent(s). Of interest, permission was not granted by the patient to contact their parent(s) for 25 of 215 parents, although in these instances the patient participated fully in the study (Table 2). Characteristics of the AYA participants are included in Table 3 . As evident, $2 / 3$ of this group was females and the majority were non-Hispanic whites $(64.6 \%)$.

\section{Conclusion and Future Directions}

The KAYAC study was established to address methodologic issues in the recruitment and participation of childhood, adolescent, and young adult cancer patients into epidemiologic studies that will promote research in this age group. Because there has been little patient-contact research conducted on this age range, the KAYAC project was intended to demonstrate the feasibility of ascertaining cases, establishing contact, and collecting data. The majority of AYA cases who agreed to participate in the study expressed both enthusiasm and interest in the research effort. Through KAYAC we have created an infrastructure by which future clinical and epidemiologic studies specific to cancer patients between 0 and 39 years old will be conducted.
Strengths of the KAYAC study include the populationbased ascertainment of cases and high participation rates of the common cancer types. Cancer registry linkage facilitates outcome measures as follow-up information may be obtained for years to come. Enrollment of parents of cases also allows for validation of some exposures which are assessed through both case and parent questionnaires and for exposure assessments during pre- and peri-natal periods. Thus, the KAYAC study allows for classification of cases in terms of a broad range of lifetime exposures.

Because the KAYAC resource is population based, adolescents and young adults as well as early onset breast cancer are represented. It is important to note that this resource has the potential to offer an in depth investigation into the uniqueness of very early onset breast cancer, as well as the heterogeneity of characteristics, etiology, and disease progression which would be important in tailoring breast cancer prevention and control in specific age groups.

The establishment of a data and biorepository designed to study cancer in kids, adolescents, and young adults has presented some challenges. Locating AYA participants was often difficult and time-consuming. While the participation rate of those for whom we had accurate contact information was quite high, of 218 cancer-registry ascertained AYA cases, 87 had inaccurate contact information and were classified as unable to contact (UTC). This could be due to several reasons, including the high mobility of this age group and difficulty in updating contact information. Even when an eligible case with contact information was identified, the efforts needed to locate, reach, and enroll them can be extensive. For the 82 AYA participants enrolled, numerous contact attempts (by phone and/or mail) were needed to obtain each individual's initial consent, schedule and conduct the interview and biospecimen collection. Additionally, while one of the strengths of our project is its minority representation and the inclusion of Spanish speakers, using bilingual interviewers and translated study documents increased study complexity and costs. 
Creating and maintaining the infrastructure required to support and sustain the KAYAC study requires a wide variety of study personnel. The study leaders include epidemiologists and an oncologist specializing in pediatric, adolescent, and young adult cancer care. Other needed staff members include research associates, a phlebotomist, a data manager, interviewers (including at least one bilingual interviewer), a statistician, and laboratory personnel.

The KAYAC project is an example of the development of an infrastructure and a resource of children and young adults with cancer and their parents for future studies. We anticipate future collaborative associations with other investigators on studies related to pediatric, adolescent and young adult cancer. Broad categories of research projects that may be facilitated or supported by this resource include the following.

(i) Investigations of biologic characteristics (such as genetic and tumor factors) of pediatric and AYA cancers that affect disease outcome (including survival, treatment response, second primary cancer, and quality of life).

(ii) Assessment of the availability and access to healthcare facilities that can enroll members of these age groups into clinical trials and determine possible barriers to access to healthcare by these patients.

(iii) Development of protocols to determine the degree of aggressiveness of cancer among the age groups included in KAYAC.

(iv) Determination of epidemiologic and environmental risk factors including family history and presence of suspect chemicals in the home environment.

(v) Development of a health literacy campaign and outreach for patients and parents.

(vi) Examination of epidemiologic characteristics (such as demographic, socioeconomic, treatment, lifestyle, pre- and peri-natal and environmental exposures) of pediatric and AYA patients that affect disease outcome (including survival, treatment response, second primary cancer, and quality of life).

In conclusion, the establishment of the KAYAC study has proven to be feasible and the participation rate of this age group was shown to be excellent. KAYAC will serve as a resource to advance knowledge and understanding of cancer incidence and outcome among this underserved group. Knowledge of the biologic, environmental, and lifestyle factors which contribute to disease progression, response to treatment, and prognosis will inform the development of targeted treatment interventions aimed at improving outcomes among this unique and vital segment of our population.

\section{Acknowledgments}

The authors wish to thank the Don and Marty Schmid Adolescent and Young Adult Cancer Fund for their support of this study. They would also like to acknowledge the Lon
V. Smith Foundation for their continued support of cancer research and this project.

\section{References}

[1] Adolescent and Young Adult Oncology Progress Review Group, "Closing the gap: research and care imperatives for adolescents and young adults with cancer," Tech. Rep., National Cancer Institute, Bethesda, Md, USA, 2006.

[2] LiveStrong Young Adult Alliance, "Closing the gap: a strategic plan," Addressing the Recommendations of the Adolescent and Young Adult Oncology Progress Review Group, National Cancer Institute, Austin, Tex, USA, 2007.

[3] A. Bleyer, A. Viny, and R. Barr, "Cancer in 15- to 29-year-olds by primary site," Oncologist, vol. 11, no. 6, pp. 590-601, 2006.

[4] A. Bleyer, T. Budd, and M. Montello, "Adolescents and young adults with cancer: the scope of the problem and criticality of clinical trials," Cancer, vol. 107, supplement 7, pp. 1645-1655, 2006.

[5] E. Ward, M. Halpern, N. Schrag, et al., "Association of insurance with cancer care utilization and outcomes," CA: Cancer Journal for Clinicians, vol. 58, no. 1, pp. 9-31, 2008.

[6] D. McGoldrick, C. Neal, and M. Whiteson, "Advocacy and adolescent/young adult cancer survivors," Pediatric Blood and Cancer, vol. 50, supplement 5, pp. 1109-1111, 2008.

[7] J. E. Haase and C. R. Phillips, "The adolescent/young adult experience," Journal of Pediatric Oncology Nursing, vol. 21, no. 3, pp. 145-149, 2004. 


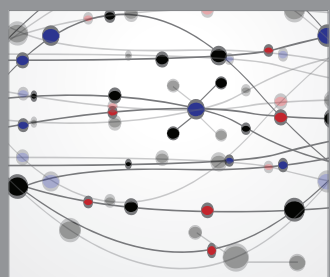

The Scientific World Journal
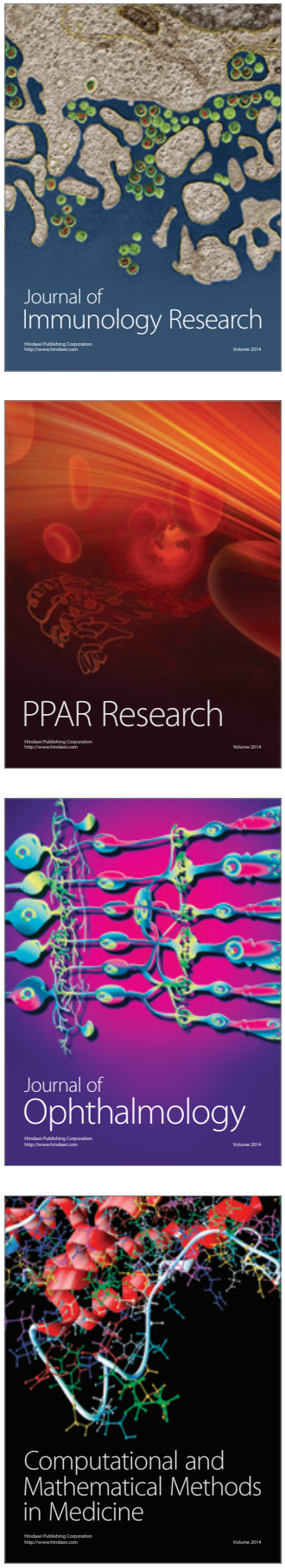

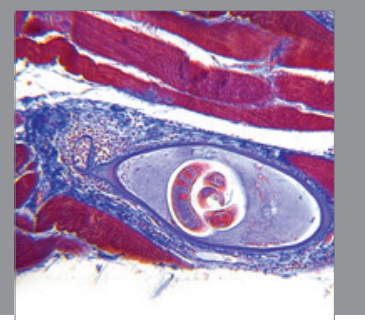

Gastroenterology

Research and Practice
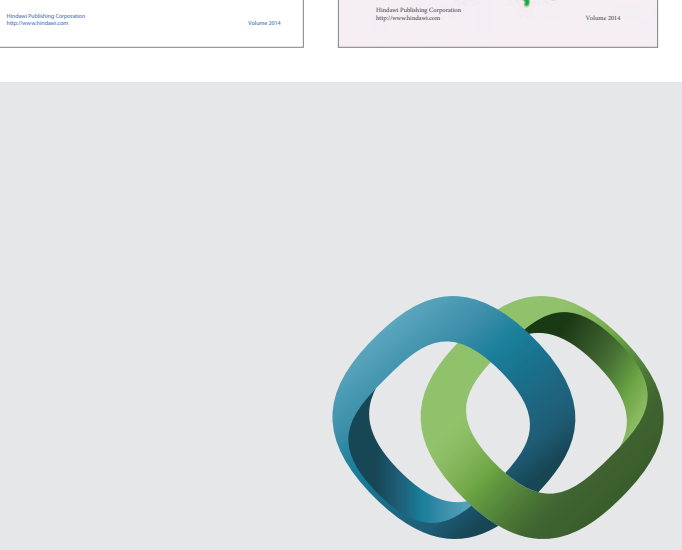

\section{Hindawi}

Submit your manuscripts at

http://www.hindawi.com
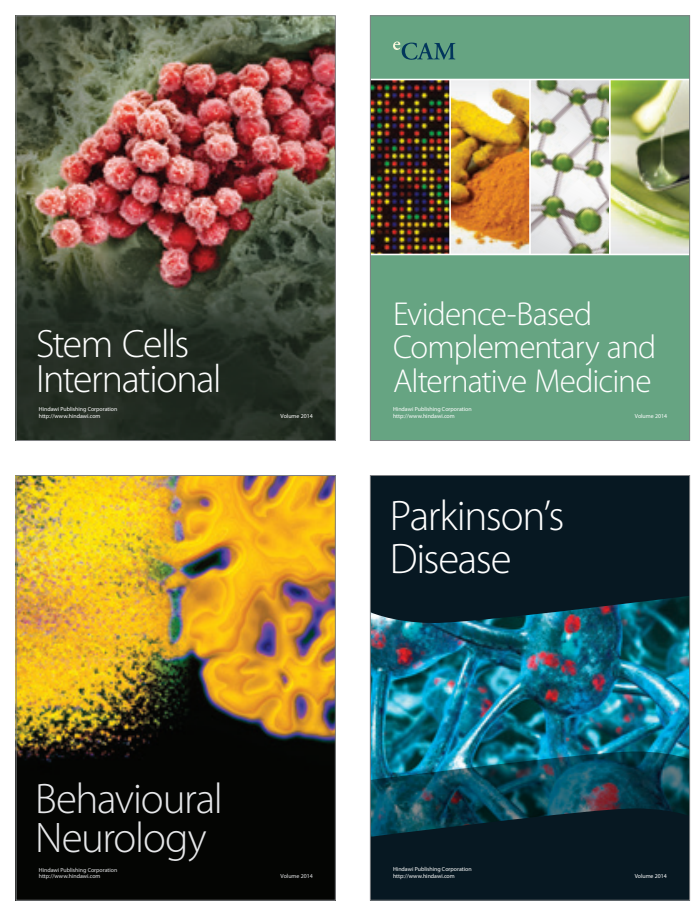

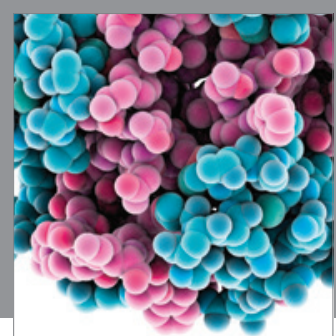

Journal of
Diabetes Research

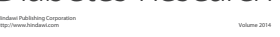

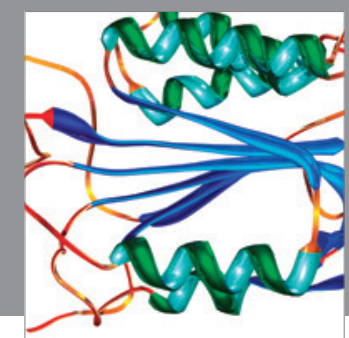

Disease Markers
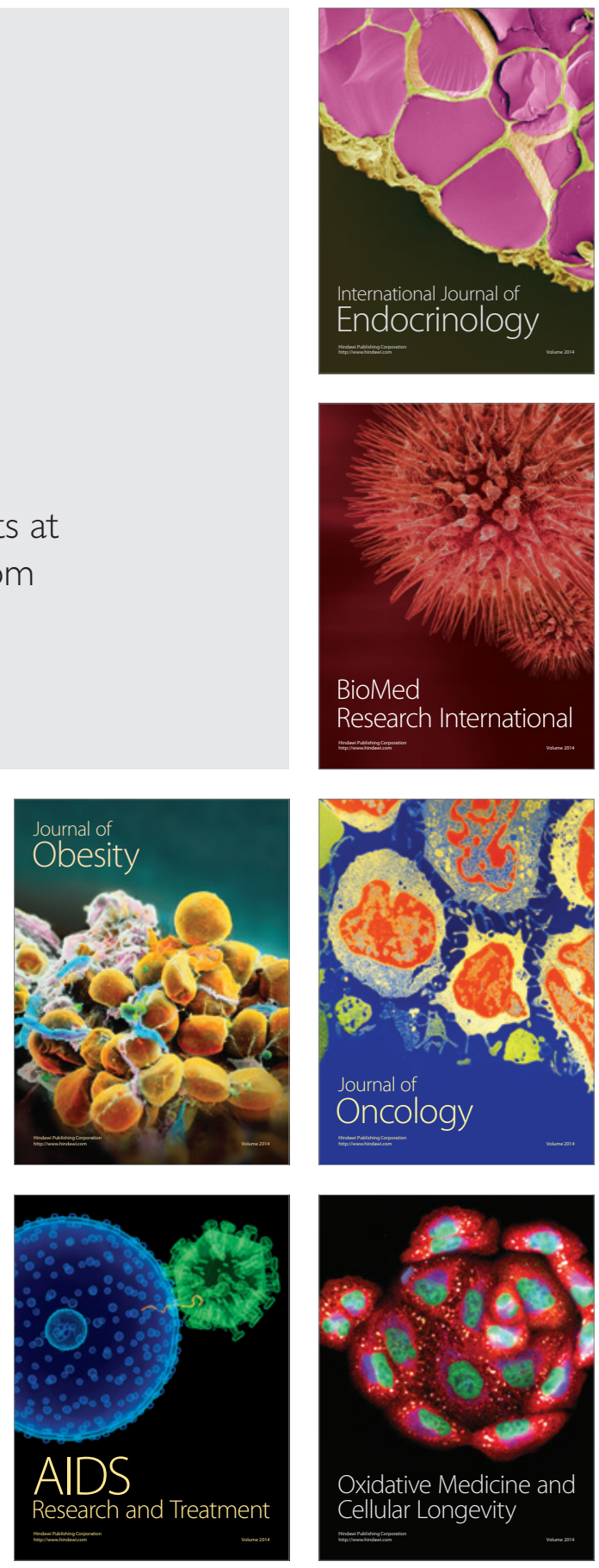